\title{
ON A LEMMA OF MARCINKIEWICZ AND ITS APPLICATIONS TO FOURIER SERIES
}

\author{
SHIGEKI YANO \\ (Received December 22, 1958)
}

1. Introduction. In a series of papers, J. Marcinkiewicz proved several theorems concerning conjugate function [6], strong summability of Fourier series [8], and integrals of Dini type [7]. The crucial point in his proofs is in the applications of a lemma concerning a metrical property of closed sets, which is stated as follows:

Let $P$ be a closed set periodic with period $2 \pi, \Delta_{n}(n=1,2, \ldots \ldots)$ be its contiguous intervals, and $\phi(x)$ be a function which is equal to $\left|\Delta_{n}\right|^{1)}$ on $\Delta_{n}$ and vanishes on $P$, then the integral

$$
\int_{-\tau}^{\pi} \frac{\varphi^{\lambda}(x+t)}{|t|^{\lambda+1}} d t
$$

is finite for almost every $x$ in $P$.

In a paper above cited [7], Marcinkiewicz introduced an integral of Dini type :

$$
\mu(f ; x)=\left(\int_{0}^{\pi} \frac{|F(x+t)+F(x-t)-2 F(x)|^{2}}{t^{3}} d t\right)^{1 / 2},
$$

where $F(x)=\int_{0}^{x} f(t) d t$, and proved that ${ }^{2)}$

$$
A\|f\|_{2} \leqq\|\mu(f)\|_{2} \leqq A\|f\|_{2} .
$$

He also conjectured and A. Zygmund [18] proved that

$$
A_{p}\|f\|_{p} \leqq\|\mu(f)\|_{p} \leqq A_{p}\|f\|_{p}, \quad 1<p<\infty,
$$

where in the left side inequality, we suppose that $\int_{0}^{2 \pi} f(x) d x=0$. The proof of Zygmund depends on "complex method"; indeed he proved that $\mu(f)$ is majorized by the Littlewood and Paley function $g^{* 3)}$.

With the aid of the lemma of Marcinkiewicz, Zygmund [17] proved that the Fourier series of an integrable function $f(x)$ is strongly summable almost

1) $|E|$ denotes the measure of a set $E$.

2) Here and hereafter, $A$ stands for an absolute positive constant, and $A_{p}$, etc. stand for constants depending only on the indicated parameters, The constants $A$ and $A_{p}$, etc. need not be the sames at different occasions.

3) For the function $g^{*}$, see [4] and [18]. 
everywhere for any order, that is, if $s_{n}(x)$ denotes the $n$-th partial sum of the Fourier series of $f(x)$, then for any $q>0$

$$
\lim _{n \rightarrow \infty} \frac{1}{n} \sum_{\nu=0}^{n}\left|s_{\nu}(x)-f(x)\right|^{q}=0
$$

almost everywhere.

In the same paper, Zygmund proved that the power series of a function $\Phi(z)$ of class $H^{\prime}, 0<\lambda<1$, is $(C, 1 / \lambda-1)$ summable almost everywhere on the circle of convergence, and conjectured the maximal inequalities for the Cesàro means: if $\sum_{n=0}^{\infty} c_{n} z^{n}$ is the power series of $\Phi(z)$, and $\sigma_{n}^{(\alpha)}(x ; \Phi)$ is its $n$-th Cesàro mean of order $\alpha$ for $z=e^{i x}$, then

$$
\int_{-\pi}^{\pi} \sup _{n}\left|\sigma_{n}^{\left(\frac{1}{\lambda}-1\right)}(x ; \Phi)\right|^{\lambda} d x \leqq A_{\lambda} \int_{-\pi}^{\pi}\left|\Phi\left(e^{i x}\right)\right|^{\lambda} \log ^{+}\left|\Phi\left(e^{i x}\right)\right| d x+A_{\lambda}
$$

and

$$
\int_{-\pi}^{\pi} \sup _{n}\left|\sigma_{n}^{\left(\frac{1}{\lambda}-1\right)}(x ; \Phi)\right|^{\lambda x} d x \leqq A_{r \lambda}\left(\int_{-\pi}^{\pi}\left|\Phi\left(e^{i x}\right)\right|^{\lambda} d x\right)^{r}, \quad 0<r<1 .
$$

Zygmund himself [20] and G. Sunouchi [15] proved (1.4) and (1.5) for $0<$ $\lambda \leqq 1 / 2$.

In the present paper, we shall prove the right side inequality of (1.3) for $1<p \leqq 2$; the maximal theorems for strong summability; and (1.4), (1.5) for $0<\lambda<1$. Our proofs are by "real method", which is a combination of the lemma of Marcinkiewicz and the lemma of F. Riesz or the maximal theorem of Hardy and Littlewood.

Our method can be applied to Walsh-Fourier series. In the last section, we consider the Paley's decomposition theorem for Walsh-Fourier series, where the lemma of Marcinkiewicz can not be applied directly, but the idea proving it also survives. ${ }^{4)}$

During the preparation of this paper, a paper of E. M. Stein [22] was published, where the results and the method of proofs have close connections with ours. However, it seems that his main objects are to extend the problems to higher dimensions, while our main aim is to show the scope of the lemma of Marcinkiewicz.

2. Fundamental lemmas. In this section, we shall state two lemmas which are fundamental in the sequels. One of them is a variant of the lemma of Marcinkiewicz stated in the introduction, and the other is a decomposition lemma of an integrable function which is essentially due to $A$.

4) In this regard, see also Fine [2] and Yano [16]. 
Zygmund. We state them, however, in slightly different forms from the original ones, so that we give the proofs here for the completeness.

Let $P$ be a closed set periodic with period $2 \pi$ on the real axis, $\Delta_{n}$ $(n=1,2, \ldots \ldots)$ be its contiguous intervals, $Q$ be the complement of $P$, $Q=\bigcup_{n=1}^{\infty} \Delta_{n}, D(x)$ be the distance from $x$ to $P .^{5)}$

For any $\lambda>0$, define :

$$
I_{\lambda}(x)=\int_{-\pi}^{\pi} \frac{D^{\lambda}(x+t)}{|t|^{\lambda+1}} d t
$$

Then a variant of the lemma of Marcinkiewicz, which is due to Ostrow and Stein [11], is stated as follows :

LEMMA 1. (1) $I_{\lambda}(x)$ is finite almost everywhere in $P$.

(2) For any $s>0$,

$$
\left|\left\{I_{\lambda}(x)>s\right\} \cap P\right| \leqq \frac{A_{\lambda}}{s}|Q| .^{\left({ }^{\prime}\right)}
$$

PROoF. (1) is due to Ostrow and Stein [11], and (2) is the immediate consequence of their proof. Here we reproduce the proof for completeness.

We can suppose that $\left|\Delta_{n}\right| \leqq 2 \pi$ for all $n$, since, if otherwise it is obvious from the periodicity that the set $P$ is of measure zero.

Since $D(x)$ vanishes on $P$, we have for $x \in P \cap[-\pi, \pi]$

$$
\begin{aligned}
I_{\lambda}(x) & =\int_{-\pi}^{\pi} \frac{D^{\lambda}(x+t)}{|t|^{\lambda+1}} d x \\
& =\int_{x-\pi}^{x+\pi} \frac{D^{\prime}(t)}{|x-t|^{\lambda+1}} d t \\
& \leqq \sum_{n}^{\prime} \int_{\Delta_{n}} \frac{D^{\lambda}(t)}{|x-t|^{\lambda+1}} d t,
\end{aligned}
$$

where the prime indicates that the summation ranges over the indices $n$ for which $\Delta_{n}$ are included in $[-3 \pi, 3 \pi]$. Then

$$
\begin{aligned}
\int_{P^{\cap}[-\pi, \pi]} I_{\lambda}(x) d x & \leqq \sum_{n}^{\prime} \int_{P \cap[-\pi, \pi]} d x \int_{\Delta_{n}} \frac{D^{\lambda}(t)}{|x-t|^{\lambda+1}} d t \\
& \leqq \sum_{n}^{\prime} \int_{\Delta_{n}} D^{\prime}(t) d t \int_{P} \frac{1}{|x-t|^{\lambda+1}} d x
\end{aligned}
$$

by Fubini's theorem. If $x \in P$ and $t \in \Delta_{n}$, then $D(t) \leqq|x-t|$, thus

5) If $P$ is empty, we define $D(x)=\infty$ for any $x$. In this case the lemma is trivial.

6) The notation $\{\ldots \ldots \ldots\}$ denotes the set which is defined by the property in the paranthese and $|E|$ denotes the measure of the set $E$, and, if $E$ is periodic, the measure of the part of $E$ contained in $[-\pi, \pi]$. 
so that

$$
\begin{aligned}
\int_{\Delta_{n}} D^{\lambda}(t) d t \int_{P} \frac{d x}{|x-t|^{\lambda+1}} & \leqq 2 \int_{\Delta_{n}} D^{\lambda}(t) d t \int_{D(t)}^{\infty} \frac{d x}{x^{\lambda+1}} \\
& =A_{\lambda} \int_{\Delta_{n}} d t=A_{\lambda}\left|\Delta_{n}\right|
\end{aligned}
$$

$$
\int_{P \cap[-\pi, \pi]} I_{\lambda}(x) d x \leqq A_{\lambda} \sum_{n}^{\prime}\left|\Delta_{n}\right|=A_{\lambda}|Q|
$$

This proves (1). Since

$$
s\left|\left\{I_{\lambda}(x)>s\right\} \cap P\right| \leqq \int_{P \cap[-\pi, \pi]} I_{\lambda}(x) d x,
$$

it follows that (2) is true.

The original form of the lemma of Marcinkiewicz can be completed as follows. Let $\phi(x)$ is equal to $\left|\Delta_{n}\right|$ on $\Delta_{n}$ and to zero on $P$, and define

$$
J_{\lambda}(x)=\int_{-\pi}^{\pi} \frac{\varphi^{\lambda}(x+t)}{|t|^{\lambda+1}} d t, \quad \lambda>0 .
$$

Then we have:

LEMMA $1^{\prime} .(1) J_{\lambda}(x)$ is finite almost everywhere in $P$.

(2) For any $s>0,\left|\left\{J_{\lambda}(x)>s\right\}\right| \leqq \frac{A_{\lambda}}{s}|Q|$.

PROOF. (1) is due to Marcinkiewicz $[6,8]$, and also it follows from (1) of Lemma 1 , since the finitenesses of $I_{\lambda}(x)$ and $J_{\lambda}(x)$ are equivalent for density points of $P$.

To prove (2), let $\Delta_{n}^{\prime}$ be the open interval concentric with $\Delta_{n}$ and having the length $3\left|\Delta_{n}\right|$. Let $Q^{\prime}$ be the union of $\Delta_{n}^{\prime}$ 's, and $P^{\prime}$ be the complement of $Q^{\prime}$. Then by a similar consideration as in the proof of lemma 1 ,

$$
\begin{aligned}
\int_{P^{\prime} \cap[-\pi, \pi]} J_{\lambda}(x) d x & =\int_{P^{\prime} \cap[-\pi, \pi]} d x \int_{-\pi}^{\pi} \frac{\varphi^{\lambda}(x+t)}{|t|^{\lambda+1}} d t \\
& \leqq \sum_{n}^{\prime} \int_{P^{\prime} \cap[-\pi, \pi]} d x \int_{\Delta_{n}} \frac{\phi^{\lambda}(t)}{|x-t|^{\lambda+1}} d t,
\end{aligned}
$$

where the prime has the same meaning as in the proof of Lemma 1. Then

$$
\begin{aligned}
\int_{P^{\prime} \cap[-\pi, \pi]} J_{\lambda}(x) d x & \leqq \sum_{n}^{\prime} \int_{\Delta_{n}} \phi^{\lambda}(t) d t \int_{P^{\prime}} \frac{d x}{|x-t|^{\lambda+1}} \\
& \leqq A_{\lambda} \sum_{n}^{\prime} \int_{\Delta_{n}} \phi^{\lambda}(t) d t \int_{\varphi(t)}^{\infty} \frac{d x}{x^{\lambda+1}} \\
& =A_{\lambda} \sum_{n}^{\prime}\left|\Delta_{n}\right|=A_{\lambda}|Q|
\end{aligned}
$$


since $|x-t| \geqq\left|\Delta_{n}\right|=\varphi(t)$ for $x \in P^{\prime}$ and $t \in \Delta_{n}$. This implies that

$$
\left|\left\{J_{\lambda}(x)>s\right\} \cap P^{\prime}\right| \leqq\left(A_{\lambda} / s\right)|Q| \text {. }
$$

Now, since $\left\{J_{\lambda}(x)>s\right\}$ is contained in the union of $\left\{J_{\lambda}(x)>s\right\} \cap P^{\prime}$ and $Q^{\prime}$, and $\left|Q^{\prime}\right| \leqq 3|Q|$, we get (2).

The following decomposition of an integrable function is due to Zygmund [19], except the explicit statement of the property (5). However, he proved this lemma in the non-periodic case, and for the proof in the present form, a slight modification is needed.

LEMMA 2. Let $f(x) \geqq 0$ be an integrable function periodic with period $2 \pi, F(x)=\int_{0}^{x} f(t) d t$

$$
M(x ; f)=\sup _{0<|t| \leq \pi} \frac{1}{t} \int_{0}^{t} f(x+u) d u,
$$

$P=P_{y}=\{M(x ; f) \leqq y\} \quad(y>0)$. Also let the set $Q$ and the distance function $D(x)$ have the same meanings as in the beginning of this section. Then

(1) $P$ is closed and

$$
|Q| \leqq \frac{A}{y} \int_{-\pi}^{\pi} f(x) d x .
$$

If $P$ is not empty, there exists a decomposition of $F(x)$ :

$$
F(x)=G(x)+H(x)
$$

and that of $f(x)$ :

$$
f(x)=g(x)+h(x)
$$

where $g(x)$ and $h(x)$ are periodic and integrable, $G(x)$ and $H(x)$ are indefinite integrals of $g(x)$ and $h(x)$, respectively, and they have the following properties: where.

(2) $g(x)=f(x)$ almost everywhere in $P$, and $0 \leqq g(x) \leqq y$ almost every-

(3) $H(x)=0$ for $x \in P$, and in particular, $\int_{\Delta_{n}} h(x) d x=0$ for every contiguous interval $\Delta_{n}$ of $P$.

$$
\begin{gathered}
\int_{-\pi}^{\pi} g(x) d x=\int_{-\pi}^{\pi} f(x) d x . \\
|H(x)| \leqq 2 y D(x) \quad \text { for any } x .
\end{gathered}
$$

PROOF. It is easy to see that $M(x)=M(x ; f)$ is periodic and lower semicontinuous, so that the set $P$ is periodic and closed. The property (1) is a consequence of the lemma of F. Riesz or the maximal theorem of Hardy and 
Littlewood; see for example, [21;pp. 241-246].

Let us suppose that $P$ is not empty. If $x \in P$, then

$$
\frac{F(x+t)-F(x)}{t} \leqq y, \quad(0<|t| \leqq \pi),
$$

hence $f(x) \leqq y$ almost everywhere in $P$.

Now, define the continuous function $G(x)$ by

$$
\begin{aligned}
G(x) & =F(x) \text { on } P \\
& =\text { linear in } \Delta_{n} \quad n=1,2, \ldots \ldots \ldots,
\end{aligned}
$$

and decompose $F(x)$ :

$$
F(x)=G(x)+H(x) .
$$

Then it is easy to see that $G(x)$ satisfies the Lipschitz condition of order 1 , and its derivative $g(x)=G^{\prime}(x)$ has the property (2) (see Zygmund $[19 ; \S 6]$ ). Hence $H(x)$ is also an indeinnite integral of an integrable $h(x)$. Since, for any $t, F(x+t)-F(x)$ is periodic with respect to the variable $x$, so is $G(x$ $+t)-G(x)$ and hence $g(x)$ is periodic. Differentiating (2.4), we get (2.5).

Since $H(x)=0$ for $x \in P$ by definition, $h(x)=0$ almost everywhere in $P$. If $\Delta_{n}=\left(a_{n}, b_{n}\right)$ is an contiguous interval of $P$, then $a_{n}, b_{n} \in P$, so that

$$
\int_{\Delta_{n}} h(x) d x=H\left(b_{n}\right)-H\left(a_{n}\right)=0,
$$

hence (3) is proved.

The property (4) follows from (3) and the periodicity of the set $P$.

Finally, we shall prove (5). For $x \in P,(5)$ is obvious, since $H(x)=D(x)$ $=0$. Let $x \in \Delta_{n}=\left(a_{n}, b_{n}\right)$ and suppose that $x$ lies to the left of the middle point of this interval. Then, by (3),

$$
\begin{aligned}
|H(x)| & =\left|H(x)-H\left(a_{n}\right)\right| \\
& \leqq\left|F(x)-F\left(a_{n}\right)\right|+\left|G(x)-G\left(a_{n}\right)\right| .
\end{aligned}
$$

Since $a_{n} \in P$, we get by (2.6)

$$
\left|F(x)-F\left(a_{n}\right)\right| \leqq y\left(x-a_{n}\right)=y D(x) .
$$

According to the property (2)

$$
\left|G(x)-G\left(a_{n}\right)\right| \leqq \int_{a_{n}}^{x} g(t) d t \leqq y\left(x-a_{n}\right)=y D(x) .
$$

In the case where $x$ lies on the right half of $\Delta_{n}$, the same estimation can be obtained, and the proof of Lemma 2 is completed.

REMARK. The conclusion (5) of Lemma 2, of course, can be replaced by

$$
|H(x)| \leqq 2 y \varphi(x)
$$

where $\phi(x)$ is the function of Marcinkiewicz, and this form of Lemma 2 
combined with Lemma $1^{\prime}$, can be used to prove the theorems in the sequel, except for Paley's theorem for decomiposition of Walsh-Fourier series, for which Lemma 2 in term of the function $D(x)$ is needed.

In the proofs of some of the theorems, we need the interpolation theorem of Marcinkiewicz and Zygmund. Since we use it only for very special cases, we restrict ourselves to the case which is needed.

Let $T$ be an operator whose domain and range are functions in $(-\pi, \pi)$. Suppose that $T$ satisfies the following two conditions :

$$
\begin{aligned}
& T(f) \text { is defined for every } f(x) \in L(-\pi, \pi) . \\
& \left|T\left(f_{1}+f_{2}\right)\right| \leqq\left|T\left(f_{1}\right)\right|+\left|T\left(f_{2}\right)\right| .
\end{aligned}
$$

For $p \geqq 1$, an operator $T$ is said to be of weak type $(p, p)$, if

$$
|\{|T(f)(x)|>y\}| \leqq \frac{A_{p}}{y^{p}} \int_{-\pi}^{\pi}|f(x)|^{p} d x
$$

for all $y>0$ and for $f(x) \in L^{p}(-\pi, \pi)$ and for $A_{p}$ independent of $y$ and $f$.

Also an operator $T$ said to be strong type $(p, p)$ if

$$
\int_{-\pi}^{\pi}|T(f)|^{p} d x \leqq A_{p} \int_{-\pi}^{\pi}|f|^{p} d x
$$

It is obvious that if $T$ is strong type $(p, p)$, then it is also of weak type $(p, p)$.

Now, the interpolation theorem of Marcinkiewicz and Zygmund [19] can be stated as follows:

LEMMA 3. Let $T$ be an operator which satisfies the condition (2.4) and (2.5). Then

(1) If $T$ is simultaneously of weak type $\left(p_{1}, p_{1}\right)$ and $\left(p_{2}, p_{2}\right), 1<p_{1}<p_{2}$ $<\infty$, then $T$ is of strong type $(p, p)$ for $p, p_{1}<p<p_{2}$.

(2) If $T$ is simultaneously of weak type $(1,1)$ and $(p, p), 1<p<\infty$, then

$$
\int_{-\pi}^{\pi}|T f| d x \leqq A \int_{-\pi}^{\pi}|f(x)| \log ^{+}|f(x)| d x+A .
$$

Finally, we state one more lemma which concerns with the integrability property of an operator of weak type (1.1):

LEMMA 4. Let $T$ be an operator which satisfies (2.4) and (2.5) and of reak type $(1,1)$, then

$$
\left(\int_{-\pi}^{\pi}|T(f)|^{r} d x\right)^{1 / r} \leqq A_{r} \int_{-\pi}^{\pi}|f(x)| d x,
$$

for any $r, 0<r<1$. 
This is known, for example, see Calderón and Zygmund [1; Theorem 4].

3. The integral of Marcinkiewicz. Marcinkiewicz [7] introduced the function (3.1) $\mu(x)=\mu(x ; f)$ :

$$
\mu(x)=\left(\int_{-\pi}^{\pi} \frac{\left|F^{\prime}(x+t)+F(x-t)-2 F(x)\right|^{2}}{t^{3}} d t\right)^{1 / 2}
$$

where $F(x)=\int_{0}^{x} f^{\prime}(t) d t$, and proved the following result :

LEMMA 5.

$$
\|\mu(f)\|_{2} \leqq A\|f\|_{2} .
$$

$\mathrm{He}$ also conjectured and Zygmund proved that

$$
A_{p}\|f\|_{p} \leqq\|\mu(f)\|_{p} \leqq A_{p}\|f\|_{p}, \quad 1<p<\infty,
$$

where in the left side we suppose that that $\int_{-\pi}^{\pi} f^{\prime}(x) d x=0$.

The proof of Zygmund for (3.2) depends on "complex method". In the present section we treat the problem by real method for the case $1 \leqq p \leqq 2$.

THEOREM 1. (1) Let $f^{\prime}(x) \in L^{1}(-\pi, \pi)$, then, for any $y>0$,

$$
|\{\mu(x ; f)>y\}| \leqq \frac{A}{y} \int_{-\pi}^{\pi}|f(x)| d x .
$$

(2) If $f(x) \in L^{p}(-\pi, \pi), 1<p \leqq 2$, then $\|\mu(f)\|_{p} \leqq A_{p}\|f\|_{p}$.

(3) If $|f(x)| \log ^{+}|f(x)| \in L^{1}(-\pi, \pi)$, then

$$
\|\mu(f)\|_{1} \leqq A \int_{-\pi}^{\pi}|f(x)| \log ^{+}|f(x)| d x+A .
$$

(4) If $f(x) \in L^{1}(-\pi, \pi)$, then

$$
\int_{-\pi}^{\pi}|\mu(f)|^{1-\epsilon} d x \leqq A_{\epsilon}\left(\int_{-\pi}^{\pi}|f(x)| d x\right)^{1-\epsilon}, \quad 0<\varepsilon<1 .
$$

PROOF. It is sufficient to prove (1), then (2) and (3) follow from Lemmas 3 and 5 ; (4) follows from Lemma 4.

Without loss of generality, we can restrict ourselves to a non-negative $f(x)$.

Given $y>0$, let us suppose the set $P$ given in Lemma 2 is not empty, and let $F(x)=G(x)+H(x)$ be the decomposition guaranteed by Lemma 2 and we shall retain the notations there. If we write

$$
\Delta_{t} F^{\prime}(x)=F(x+t)+F(x-t)-2 F(x)
$$

then

$$
\Delta_{t} F(x)=\Delta_{t} G(x)+\Delta_{t} H(x)
$$

so that we have 


$$
\mu(x ; f) \leqq \mu(x ; g)+\mu(x ; h) .
$$

Since $0 \leqq g(x) \leqq y$ almost everywhere by (2) of Lemma $2, g(x) \in L^{2}$. Hence

$$
\int_{-\pi}^{\pi} \mu(g)^{2} d x \leqq A \int_{-\pi}^{\mu} g(x)^{2} d x
$$

by Lemma 5 , and thus

$$
\int_{-\pi}^{\pi} g(x)^{2} d x \leqq y \int_{-\pi}^{\pi} g(x) d x=y \int_{-\pi}^{\pi} f(x) d x .
$$

This, together with (3.3), implies that

$$
|\{\mu(x ; g)>y / 2\}| \leqq \frac{A}{y} \int_{-\pi}^{\pi} f(x) d x .
$$

We next turn to $\mu(x ; h)$.

Since $H(x)=0$ for $x \in P$ by Lemma 2 , if $x \in P$, then

$$
\begin{aligned}
\mu(x ; h) & =\left(\int_{0}^{\pi} \frac{|H(x+t)+H(x-t)-2 H(x)|^{2}}{t^{3}} d t\right)^{1 / 2} \\
& \leqq\left(\int_{-\pi}^{\pi} \frac{|H(x+t)|^{2}}{t^{3}} d t\right)^{1 / 2} .
\end{aligned}
$$

Since $|H(x)| \because 2 y D(x)$ by Lemma 2 ,

$$
\mu(x ; h) \leqq 2 y\left(\int_{-\pi}^{\pi} \frac{D^{2}(x+t)}{t^{3}} d t\right)^{1 / 2}
$$

Let $\mathfrak{F}$ denote the set $\left\{I_{2}(x) \leqq 1 / 4\right\}$, where $I_{2}(x)$ is defined by (2. 1$)$, then for $x \in P \cap \mathfrak{F}$,

$$
\mu(x ; h) \leqq y / 2 .
$$

Hence the set $\{\mu(x ; h)>y / 2\}$ is contained in the complement of the set $P \cap \mathfrak{F}$, which is contained in the union of $Q$ and the set $\mathbb{E}=\left\{I_{2}(x)>1 / 4\right\}$. Since

$$
|Q| \leqq \frac{A}{y} \int_{-\pi}^{\pi} f(x) d x, \quad|ほ| \leqq A|Q|
$$

by Lemma 2 and Lemma 1 , it follows that

$$
|\{\mu(x ; h)>y / 2\}| \leqq \frac{A}{y} \int_{-\pi}^{\pi} f(x) d x .
$$

This, together with (3.4), implies (1).

If the set $P$ is empty, (1) is trivial for such $y$ by virtue of (1) of Lemma 2 , and the proof of Theorem 1 is completed.

More generally, the same reasoning gives the following theorem:

THEOREM 2. Let 


$$
\mu_{q}(x ; f)=\left(\int_{0}^{\pi} \frac{|F(x+t)+F(x-t)-2 F(x)|^{q}}{t^{q+1}} d t\right)^{1 / q}, \quad 2 \leqq q<\infty,
$$

where $F(x)=\int_{0}^{x} f(t) d t$. Then

$$
\left|\left\{\mu_{q}(x ; f)>y\right\}\right| \leqq \frac{A_{q}}{y} \int_{-\pi}^{\pi}|f(x)| d x .
$$

The analogous inequality (3.2) for the function $\mu_{q}(x ; f)$ is known, see Marcinkiewicz [7] and G. Sunouchi [13].

Another extention to non-periodic case of the integral of Marcinkiewicz will be discussed in $\S 5$.

4. Strong summability of Fourier series. Let $f(x)$ be an integrable function, and $s_{n}(x)$ be the $n$-th partial sum of its Fourier series. We say that the Fourier series of $f(x)$ is strongly summable in order $r$ or summable $H_{r}$ at a point $x$ if

$$
\lim _{n \rightarrow \infty} \frac{1}{n+1} \sum_{\nu=0}^{n}\left|s_{\nu}(x)-f(x)\right|^{r}=0 .
$$

Marcinkiewicz [8] proved by the use of Lemma 1', that the Fourier series of an integrable function is summable $\mathrm{H}_{2}$ almost everywhere, and refining his method, Zygmund [17] extended the result of Marcinkiewicz for any $r>0$.

Let us write

$$
T_{r}(x ; f)=T_{r}(x)=\left\{\sup _{u \leqq n<\infty} \frac{1}{n+1} \sum_{\nu=0}^{n}\left|s_{\nu}(x)\right|^{r}\right\}^{1 / r},
$$

then it is known that, for any $r>0$

$$
\int_{-\pi}^{\pi} T_{r}(f)^{p} d x \leqq A_{p r} \int_{-\pi}^{\pi}|f(x)|^{p} d x, \quad 1<p<\infty,{ }^{7)}
$$

and

$$
\int_{-\pi}^{\pi} T_{r}(f) d x \leqq A_{r} \int_{-\pi}^{\pi}|f(x)| \log ^{2}(1+|f(x)|) d x+A_{r}{ }^{8} .
$$

Now, we shall prove that $T_{r}(x ; f)$ is essentially majorized by the maximal function $M(x ; f)$.

THEOREM 3. Let $f(x)$ be integrable and $T_{r}(x ; f)$ be defined by (5.1). Then for any $r>0$,

$$
\left|\left\{T_{r}(x ; f)>y\right\}\right| \leqq A_{r}\left|\left\{M(x ; f)>A_{r} y\right\}\right| .
$$

7) See Sunouchi-Yano [14].

8) See Marcinkiewicz [10]. 
(2) If $f(x) \in L^{p}, 1<p<\infty$, then $\int_{-\pi}^{\pi} T_{r}(x)^{p} d x \leqq A_{p r} \int_{-\pi}^{\pi}|f(x)|^{p} d x$.

(3) If $|f(x)| \log ^{+}|f(x)| \in L^{1}$, then

$$
\int_{-\pi}^{\pi} T_{r}(x) d x \leqq A_{r} \int_{-\pi}^{\pi}|f(x)| \log ^{+}|f(x)| d x+A_{r} .
$$

(4) If $f(x) \in L^{1}$, then $\int_{-\pi}^{\pi} T_{r}(x)^{1-\varepsilon} d x \leqq A_{r \epsilon}\left(\int_{-t}^{\pi}|f(x)| d x\right)^{1-\varepsilon}, 0<\varepsilon<1$.

PROOF. We can restrict ourselves to $f(x) \geqq 0$ and for $r>2$, since $T_{r}(x ; f)$ is increasing with $r$. Then the theorem is a consequence of the following two lemmas. Given $y>0$, the set $P, Q$ are those given in Lemma 2 and the functions $\varphi(x)$ and $D(x)$ and $I_{\lambda}(x)$ and $J_{\lambda}(x)$ ars those defined correspondingly to them.

LEMMA 6. If $f(x)$ is integrable, $1<p<2,1 / p+1 / q=1$, then for $x \in P$

$$
T_{q}(x ; f) \leqq A_{q}\left\{y^{p}+y^{p-1} \int_{-\pi}^{\pi} \frac{\phi^{p-1}(x+t) f(x+t)}{|t|^{p}} d t\right\}^{1 / p}+A_{q} M(x ; f) .
$$

This is due to Zygmund [17]. We can replace $\varphi(x)$ by $D(x)$ in (5.4). Indeed, the function $\varphi(x)$ in (5.4) was introduced by the use of a lemma of Hardy and Littlewood [3]: Let $f(x) \geqq 0$ be integrable and $f(\rho, x)$ be its Poisson integral. Then for $x \in P$

$$
f(\rho, x+t) \leqq A y\left(1+\frac{|t|}{1-\rho}\right) .
$$

It is easy to see that (5.5) may be stated as follows:

$$
f(\rho, x+t) \leqq A y\left(1+\frac{\varphi(x+t)}{1-\rho}\right)
$$

or

$$
f(\rho, x+t) \leqq A y\left(1+\frac{D(x+t)}{1-\rho}\right) .
$$

If we use (5.7) instead of (5.6) in the proof in [17], we can replace $\varphi(x)$ by $D(x)$ in (5.4).

We can prove the theorem by either form of lemma 6. However it is convienient here to use it in the present form (5.4).

LEMMA 7. Let us write $\mathfrak{F}_{p}=\left\{J_{p}(x) \leqq 1\right\}$. Then for almost all $x \in \mathfrak{F}_{p-1}$ $\cap \mathfrak{F}_{p} \cap P$,

$$
\tau(x ; f)=\int_{-\pi}^{\pi} \frac{\phi^{p-1}(x+t) f(x+t)}{|t|^{p}} d t \leqq A_{p} y .
$$


PROOF. Since $f^{\prime}(x) \geqq 0$ and $\varphi^{\prime}(x)=0$ in $P$ and we can suppose that, for any contiguous interval $\Delta_{n}$ of $P,\left|\Delta_{n}\right|<2 \pi$, it follows that, for $x \in$ $[-\pi, \pi]$,

$$
\tau(x ; f) \leqq \sum_{n}^{\prime} \int_{\Delta_{n}} \frac{\varphi^{p-1}(t) f(t)}{|x-t|^{p}} d t=\tau_{1}(x ; f),
$$

where the prime indicates that the summation ranges over those $n$ for which $\Delta_{n}$ are contained in the interval $[-3 \pi, 3 \pi]$. Let $f(x)=g(x)+h(x)$ be the decomposition given by Lemma 2 , then

$$
\tau_{1}(x ; f)=\tau_{1}(x ; g)+\tau_{1}(x ; h) .
$$

Since $0 \leqq g(x) \leqq y$ almost everywhere

$$
\begin{aligned}
\tau_{1}(x ; g) & \leqq y \sum_{n}^{\prime} \int_{\Delta_{n}} \frac{\varphi^{p-1}(t)}{|x-t|^{p}} d t \\
& \leqq y \int_{-3 \pi}^{3 \pi} \frac{\varphi^{p-1}(t)}{|x-t|^{p}} d t=A_{p} y \int_{-\pi}^{\pi} \frac{\varphi^{p-1}(x+t)}{|t|^{p}} d t \\
& =A_{p} y J_{p-1}(x) .
\end{aligned}
$$

Hence for $x \in \mathfrak{F}_{p-1} \cap P$,

$$
\tau_{1}(x ; g) \leqq A_{p} y .
$$

We next estimate $\tau_{1}(x ; h)$. Let $\Delta_{n}=\left(a_{n}, b_{n}\right)$, then

$$
\tau_{1}(x ; h)=\sum_{n}^{\prime} \int_{x_{n}}^{j_{n}} \frac{\phi^{p-1}(t) h(t)}{|x-t|^{p}} d t .
$$

Since $\varphi(t)=\left|\Delta_{n}\right|$ on $\Delta_{n}$,

$$
\int_{a_{n}}^{b_{n}} \frac{\varphi^{p_{-1}}(t) h(t)}{|x-t|^{p}} d t=\left|\Delta_{n}\right|^{p_{-1}} \int_{a_{n}}^{b_{n}} \frac{h(t)}{|x-t|^{p}} d t .
$$

Now, by integration by parts, if $x \in P$ coincides neither $a_{n}$ nor $b_{n}$, then

$$
\left|\int_{a_{n}}^{b_{n}} \frac{h(t)}{|x-t|^{p}} d t\right|=p\left|\int_{i_{n}}^{b_{n}} \frac{H(t)}{|x-t|^{p_{+1}}} d t\right|,
$$

where $H(t)$ is an indefinite integral of $h(x)$ having the property (3) and (5) of Lemma 2. Thus

$$
\begin{aligned}
\tau_{1}(x ; h) & \leqq \sum_{n}^{\prime}\left|\Delta_{n}\right|^{p_{-1}} \int_{\Delta_{n}} \frac{|H(t)|}{|x-t|^{p_{+1}}} d t \\
& =\sum_{n}^{\prime} \int_{\Delta_{n}} \frac{\phi^{p_{-1}}(t)|H(t)|}{|x-t|^{p_{+1}}} d t \\
& \leqq 2 y \sum_{n}^{\prime} \int_{\Delta_{n}} \frac{\phi^{p}(t)}{|x-t|^{p_{1}}} d t
\end{aligned}
$$

since $|H(t)| \leqq 2 y D(t)$ and $D(t) \leqq \varphi(t)$. Therefore, if $x \in \mathfrak{F}_{p} \cap P$ and is not an end point of any $\Delta_{n}$, then 


$$
\tau_{1}(x ; h)=A_{p} y \int_{-3 \pi}^{3 \pi} \frac{\varphi^{p}(x+t)}{|t|^{p+1}} d t=A_{p} J_{p}(x) y \leqq A_{p} y .
$$

From (5.8) and (5.9), it follows that, for $x \in \mathfrak{F}_{p-1} \cap \mathfrak{F}_{p} \cap P$, which is not an end point of any $\Delta_{n}, \tau(x ; f) \leqq A_{p} y$. This completes the proof of Lemma 7.

Now, we return to the proof of the theorem. According to Lemmas 6 and 7, it follows that, for almost all $x \in \mathfrak{F}_{p-1} \cap \mathfrak{F}_{p} \cap P$,

$$
T_{q}(x ; f) \leqq A_{q} y+A_{q} M(x ; f) .
$$

Hence, if we denote $\mathfrak{F}_{p}=\left\{J_{p}(x)>1\right\}$, then

$$
\left\{T_{q}(x ; f)>A_{q} y\right\} \subset \mathfrak{F}_{p-1} \cup \mathfrak{F}_{p} \cup Q,
$$

so that

$$
\left|\left\{T_{q}(x ; f)>A_{q} y\right\}\right| \leqq\left|\mathfrak{F}_{p-1}\right|+\left|\xi_{p}\right|+|Q| .
$$

Since $\left|\mathfrak{F}_{p-1}\right| \leqq A_{p}|Q|,\left|\mathfrak{F}_{p}\right| \leqq A_{p}|Q|$ by Lemma $1^{\prime}$, we obtain the conclusion (1) of Theorem 3.

The conclusions (2)-(4) are the easy consequences of (1). Indeed, it is easy to see that, for any measurable $g(x) \geqq 0$,

$$
\int_{-\pi}^{\pi} g(x)^{p} d x=\int_{0}^{\infty}\left|\left\{g(x)^{p}>y\right\}\right| d y=p \int_{0}^{\infty}|\{g(x)>y\}| y^{p-1} d y \quad(p>0) .
$$

Hence, according to (1)

$$
\begin{aligned}
\int_{-\pi}^{\pi} T_{r}(x)^{p} d x & =\int_{0}^{\infty}\left|\left\{T_{r}(x)>y\right\}\right| y^{p_{-1}} d y \\
& \leqq A_{p r} \int_{0}^{\infty}|\{M(x)>y\}| y^{p_{-1}} d y \\
& =A_{p r} \int_{-\pi}^{\pi} M(x)^{p} d x .
\end{aligned}
$$

Then, from the maximal theorem of Hardy and Littlewood (see, for example, [21; p. 246]), the desired conclusions follow.

5. Cesàro summability of power series. Let

$$
\Phi(z)=\sum_{n=0}^{\infty} c_{n} z^{n}
$$

be a function of class $H^{\lambda}, \lambda>0$, that is, $\Phi(z)$ is regular for $|z|<1$ and

$$
\int_{-\pi}^{\pi}\left|\Phi\left(r e^{i x}\right)\right|^{\lambda} d x=O(1)
$$

as $r \rightarrow 1$.

Let $\sigma_{n}^{(\alpha)}(x)$ be the $n$-th Cesàro mean of order $\alpha$ of the series 


$$
\sum_{n=0}^{\infty} c_{n} e^{i n x}
$$

and set

$$
\sigma^{(\alpha)}(x)=\sup _{0 \leqq n<\infty}\left|\sigma_{n}^{(\alpha)}(x)\right| .
$$

Zygmund [17] proved that if $\Phi(z) \in H^{\lambda}, 0<\lambda<1$, then the series (5.2) is $(C, 1 / \lambda-1)$ summable to $\Phi\left(e^{i x}\right)$ almost everywhere, and also he conjectured that

$$
\int_{-\pi}^{\pi} \sigma^{(1 / \lambda-1)}(x)^{\lambda} d x \leqq A^{\lambda} \int_{-\pi}^{\pi}\left|\Phi\left(e^{i x}\right)\right|^{\lambda} \log ^{+}\left|\Phi\left(e^{i x}\right)\right| d x+A_{\lambda}
$$

and

$$
\int_{-\pi}^{\pi} \sigma^{(1 / \lambda-1)}(x)^{r \lambda} d x \leqq A_{\lambda r}\left(\int_{-\pi}^{\pi}\left|\Phi\left(e^{i x}\right)\right|^{\lambda} d x\right)^{r}, \quad 0<r<1 .
$$

Zygmund himself [20] and Sunouchi [15] have proved (5.4) and (5.5) for $0<\lambda \leqq 1 / 2$.

We shall here give the proof of (5.4) and (5.5) for $0<\lambda<1$ by an application of Lemma 1'and Lemma 2.

THEOREM 4. Let $\Phi(z) \in H^{\lambda}, 0<\lambda<1$, and $\sigma^{(\alpha)}(x)$ is defined by (5.3). Then for any $y>0$,

(1) $\left|\left\{\sigma^{(1 / \lambda-1)}(x)^{\lambda}>A_{\lambda} y\right\}\right| \leqq A_{\lambda}\left|\left\{M\left(x ;\left|\Phi\left(e^{i x}\right)\right|^{\lambda}\right)>y\right\}\right|$.

(2) We have (5.4) and (5.5) for $0<\lambda<1$.

PROOF. It is sufficient to prove (1), then (2) follows from the same argument as in the last part of the proof of Theorem 3 .

Let us write $f(x)=\left|\Phi\left(e^{i x}\right)\right|^{\lambda}$, then $f(x) \in L^{1}$. We apply Lemma 2 for $f(x)$ and retain the notations there and in the proof of Theorem 3 . The conclusion (1) for the case $1 / 2 \leqq \lambda<1$ is a consequence of Lemma 7 and the following lemma.

LEMMA $8^{9)}$. If $\Phi(z) \in H^{\lambda}, 1 / 2 \leqq \lambda<1$, then for $x \in P$

$$
\left|\sigma^{(\alpha)}(x ; \Phi)\right|^{\lambda} \leqq A_{\lambda} y\left\{1+\frac{1}{y} \int_{-\pi}^{\pi} \frac{\varphi^{\alpha}(x+t)\left|\Phi\left(e^{i(x+t)}\right)\right|^{\lambda}}{|t|^{\alpha+1}} d t\right\}
$$

where $\alpha=1 / \lambda-1$.

This is due to Zygmund [17].

The proof of the theorem is now immediate. According to Lemmas 7 and 8 , we get

$$
\left|\sigma^{(\alpha)}(x)\right|^{\lambda} \leqq A_{\lambda} y, \quad(\alpha=1 / \lambda-1),
$$

9) We can also replace $\rho(x)$ by $D(x)$ in this lemma. 
for almost almost all $x \in \mathfrak{F}_{\alpha} \cap \mathfrak{F}_{\alpha+1} \cap P$ and hence

$$
\left\{\sigma^{(\alpha)}(x)^{\lambda}>A_{\lambda} y\right\} \subset \xi_{\alpha} \cup \xi_{\alpha+1} \cup Q .
$$

This implies (1).

If $\Phi(z) \in H^{\lambda}, 1 / 4 \leqq \lambda<1 / 2$, we can suppose $\Phi(z) \neq 0$ for $|z|<1$ and set $\Phi(z)=\Psi^{2}(z), \Psi(z) \in H^{2 \lambda}$, and thus

$$
s_{n}^{(\alpha)}(x ; \Phi)=\sum_{\nu=0}^{n} s_{\nu}^{\frac{1}{2}(\alpha-1)}(x ; \Psi) s_{n-\nu}^{\frac{1}{2}(\alpha-1)}(x ; \Psi),
$$

where $s_{n}^{(\alpha)}(x ; \Phi)$ is the $n$-th Cesàro sum of the power series of $\Phi(z)$ at $z=e^{i x}$. Hence

$$
\left|\sigma^{(\alpha)}(x ; \Phi)\right| \leqq\left|\sigma^{\frac{1}{2}(\alpha-1)}(x ; \Psi)\right|^{2} .
$$

Now, since $1 / 2 \leqq 2 \lambda<1$, we have by the preceding case

$$
\begin{aligned}
\left|\left\{\left[\sigma^{\left(\frac{1}{\lambda}-1\right)}(x ; \Phi)\right]^{\lambda}>y\right\}\right| & \leqq\left|\left\{\left[\sigma^{\left(\frac{1}{2 \lambda}-1\right)}(x ; \Psi)\right]^{2 \lambda}>y\right\}\right| \\
& \leqq A_{\lambda}\left|\left\{M\left(x ;|\Psi|^{2 \lambda}\right)>y\right\}\right| \\
& =A_{\lambda}\left|\left\{M\left(x ;|\Phi|^{\lambda}\right)>y\right\}\right|,
\end{aligned}
$$

and this implies (1) for $1 / 4 \leqq \lambda<1 / 2$.

The same argument can be applied to the case $1 / 8 \leqq \lambda<1 / 4$, and so on, and the proof of Theorem 4 is completed.

6. Real analogues of the functions of Littlewood and Paley, and of Lusin. Let $f(x)$ be an integrable function periodic with period $2 \pi$, and $\phi\left(r e^{i x}\right)$ be the function regular for $|z|<1$ and whose real part is the Poisson integral of $f(x)$. Littlewood and Paley [4] introduced the functions $g$ and $g^{*}$ :

$$
\begin{gathered}
g(x)=g(x ; f)=\left\{\int_{0}^{1}(1-r)\left|\boldsymbol{\varphi}^{\prime}\left(r e^{i x}\right)\right|^{2} d r\right\}^{1 / 2}, \\
g^{*}(x)=g^{*}(x ; f)=\left\{\int_{0}^{1}(1-r) d r \frac{1}{\pi} \int_{-\pi}^{\pi}\left|\boldsymbol{\varphi}^{\prime}\left(r e^{i(x+t)}\right)\right|^{2} P(r, t) d t\right\}^{1 / 2},
\end{gathered}
$$

where $P(r, t)=\left(1-r^{2}\right) / 2\left(1+2 r \cos t+r^{2}\right)$ is the Poisson kernel. Their main results are

$$
\begin{array}{ll}
\|g(f)\|_{p} \leqq A_{p}\|f\|_{p}, & 1<p<\infty, \\
\left\|g^{*}(f)\right\|_{p} \leqq A_{p}\|f\|_{p}, & 1<p<\infty
\end{array}
$$

where (6.4) was first proved for the case $p=2,4,6, \ldots \ldots$ by th $\lrcorner m$ and later Zygmund completed for the general case $1<p<\infty$. Moreover, Zygmund has recently proved that, if $\phi(z) \in H$, then

$$
\left\|g^{*}(\varphi)\right\| \leqq A \int_{-\pi}^{\pi}\left|\varphi\left(e^{i x}\right)\right| \log ^{+}\left|\varphi\left(e^{i x}\right)\right| d x+A
$$




$$
\int_{-\pi}^{\pi}\left|g^{*}(\varphi)\right|^{1-\epsilon} d x \leqq A_{\epsilon}\left(\int_{-\pi}^{\pi}\left|\varphi\left(e^{i x}\right)\right| d x\right)^{1-\epsilon}, \quad 0<\varepsilon<1 .
$$

Also, N. Lusin [5] introduced the function

$$
S(x)=S(x ; \phi)=\left(\iint_{\Omega_{\gamma}(x)}\left|\phi^{\prime}\left(r e^{i t}\right)\right|^{2} d \omega\right)^{1 / 2},
$$

where $\phi(z)$ is regular for $|z|<1, \Omega_{\gamma}(x)$ is the part of the unit circle limited by the two chords through $e^{i x}$ at angle $\gamma\left(0<\gamma<\frac{\pi}{2}\right)$ to the radius and the parpendiculars upon them from the origin, and $d \omega$ is the Euclidean area element.

Marcinkiewicz and Zygmund [9] proved that

$$
\|S(\varphi)\|_{p} \leqq A_{p \gamma}\|\varphi\|_{p}, \quad 0<p<\infty,
$$

from which it follows, by a theorem of M. Riesz, that

$$
\|S(\varphi)\|_{p} \leqq A_{p \gamma}\|f\|_{p}, \quad 1<p<\infty .
$$

Moreover, it is known [18] that $g^{*}(\phi)$ is a majorant of $S(\phi)$, so that the relation (6.9) is a consequence of (6.4).

In the present section we shall consider the real analogues of these functions.

The real analogue of the function $g$ was first considered by Zygmund [18]. Let $f(r, x)$ be the Poisson integral of $f(x)$, and define

$$
\begin{gathered}
k(x ; f)=\left\{\int_{0}^{1} r^{-2}(1-r)\left|f_{x}(r, x)\right|^{2} d r\right\}^{1 / 2}, \\
k^{*}(x ; f)=\left\{\int_{0}^{1} r^{-2}(1-r) d r \frac{1}{\pi} \int_{-\pi}^{\pi}\left|f_{x}(r, x+t)\right|^{2} P(r, t) d t\right\}^{1 / 2} .
\end{gathered}
$$

It is easily seen that $k(x: f) \leqq 2 k^{*}(x: f) \leqq 2 g^{*}(x ; f)$.

Zygmund proved that

$$
k(x ; f) \leqq A \mu(x ; f),
$$

where $\mu(x ; f)$ is the function of Marcinkiewicz, so that, according to Theorem 1 , we have the following theorem.

THEOREM 5. (1) For any $y>0$,

$$
\mid\left\{k(x ; f)>y\left|<\frac{A_{\gamma}}{y} \int_{-\pi}^{\pi}\right| f(x) \mid d x .\right.
$$

(2) If $f(x) \in L^{p}, 1<p \leqq 2$, then $\|k(f)\|_{p} \leqq A_{p}\|f\|_{p}$.

We could not succeed in proving by real method the analoguous inequality for the function $k^{*}(f)$, but we can treat the real analogue of the function of Lusin by the preceding method. 
Let

$$
U(x ; f)=\left(\iint_{\Omega \gamma(x)} r^{-2}\left|f_{t}(r, t)\right|^{2} d r d t\right)^{1 / 2}
$$

Then we have:

THEOREM 6. (1) For any $y>0$,

$$
|\{U(x ; f)>y\}| \leqq \frac{A_{\gamma}}{y} \int_{-\pi}^{\pi}|f(x)| d x .
$$

(2) If $f(x) \in L^{p}, 1<p \leqq 2$, then $\quad\|U(f)\|_{p} \leqq A_{p \gamma}\|f\|_{p}$. lows.

PROOF. Following the method of Zygmund [18], we can argue as fol-

We can suppose that $f(x) \geqq 0$.

If $f(x)=g(x)+h(x)$ is the decomposition given by Lemma 2 , then

$$
U(x ; f) \leqq U(x ; g)+U(x ; h) .
$$

It is easily seen by a direct computation that

$$
\|U(x ; g)\|_{2} \leqq A_{\gamma}\left\|g^{\prime}\right\|_{2} \leqq A_{\gamma} y\|f\|_{1}
$$

from which it follows as before that

$$
|\{U(x ; g)>y\}| \leqq \frac{A_{\gamma}}{y} \int_{-\pi}^{\pi} f(x) d x .
$$

We now turn to $U(x ; h)$. Since $\int_{-\pi}^{\pi} h(x) d x=0$ by (4) of Lemma 2 , we have by integration by parts

$$
\begin{aligned}
h_{x}(r, x+t) & =\frac{\partial}{\partial x} \int_{-\pi}^{\pi} h(u) P(r, x+t-u) d u \\
& =\int_{-\pi}^{\pi} h(u) \frac{\partial}{\partial u} P(r, x+t-u) d u \\
& =\int_{-\pi}^{\pi} H(x+u) \frac{\partial^{2}}{\partial u^{2}} P(r, t-u) d u .
\end{aligned}
$$

Now

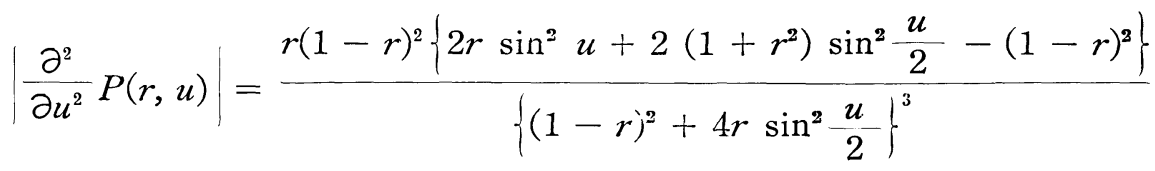

$$
\begin{aligned}
& \leqq A \frac{r \delta\left(\delta^{2}+u^{2}\right)}{\left\{\delta^{2}+u^{2}\right\}^{3}}=\frac{A r \delta}{\left\{\delta^{2}+u^{2}\right\}^{2}}
\end{aligned}
$$

where we set $\delta=1-r$. Hence 


$$
\begin{aligned}
U^{2}(x ; h) & \leqq A \iint_{\Omega \gamma(\boldsymbol{X})} r^{-2}\left|h_{t}(r, t)\right|^{2} d r d t \leqq A \iint_{\Omega \gamma(0)} r^{-2}\left|h_{x}(r, x+t)\right|^{2} d r d t \\
& =A_{\gamma} \int_{0}^{1} \delta^{2} d r \int_{|t| \leqq \alpha \delta} d t\left\{\int_{-\pi}^{\pi} H(x+u) \frac{d u}{\left\{\delta^{2}+(t-n)^{2}\right\}}\right\}^{2},
\end{aligned}
$$

since $0 \leqq|t| \leqq \alpha \delta\left(\alpha=\alpha_{\gamma}\right)$ for the point $r e^{i t}$ in the domain $\Omega_{\gamma}(0)$. Then by Schwarz' inequality,

$$
\begin{aligned}
U^{2}(x ; h) & \leqq A_{\gamma} \int_{0}^{1} \delta^{2} d r \int_{|t| \leqq \alpha \delta} d t\left\{\int_{-\pi}^{\pi} \frac{H^{2}(x+u)}{\left\{\delta^{2}+(t-u)^{2}\right\}^{3}} d u\right\}\left\{\int_{-\pi}^{\pi} \frac{d u}{\delta^{2}+(t-u)^{2}}\right\} \\
& =A_{\gamma} \int_{0}^{1} \delta d r \int_{-\pi}^{\pi} H^{2}(x+u) d u \int_{|t| \leqq \alpha \delta \delta} \frac{d t}{\left\{\delta^{2}+(t-u)^{2}\right\}^{3}} .
\end{aligned}
$$

We shall estimate the inner double integral. For this purpose, we divide the rectanagle $0 \leqq|u| \leqq \pi, 0 \leqq|t| \leqq \alpha \delta$ into four parts : $0 \leqq \pm u \leqq \pi, 0$ $\leqq \pm t \leqq \alpha \delta$. Since the arguments are similar for any parts, we consider the integral for $0 \leqq u \leqq \pi, 0 \leqq t \leqq \alpha \delta$. Then

$$
\begin{aligned}
& \int_{0}^{\pi} H^{2}(x+u) d u \int_{0}^{\alpha \delta} \frac{d t}{\left\{\delta^{2}+(t-u)^{2}\right\}^{3}} \\
& \leqq \int_{0}^{2 \alpha \delta} H^{2}(x+u) d u \int_{0}^{\alpha \delta} \frac{d u}{\left\{\delta^{2}+(t-u)^{2}\right\}^{3}} \\
& \quad+\int_{2 \alpha \delta}^{\pi} H^{2}(x+u) d u \int_{0}^{\frac{1}{2} u} \frac{d t}{\left\{\delta^{2}+(t-u)^{2}\right\}^{3}} .
\end{aligned}
$$

If $0 \leqq u \leqq 2 \alpha \delta, 0 \leqq t \leqq \alpha \delta$, then $(u-t)^{2} \leqq A_{\gamma} \delta^{2}$, so that the first term in the right side of (6.16) is less than

$$
A_{\gamma} \delta^{-5} \int_{0}^{2 \alpha \delta} H^{2}(x+u) d u \text {. }
$$

Also, if $2 \alpha \delta \leqq u \leqq \pi, 0 \leqq i \leqq \frac{1}{2} u$, then $u-t \geqq \frac{1}{2} u \geqq \alpha \delta$, hence the second term (6.16) is less than

$$
A_{\gamma} \int_{2 \alpha \delta}^{\pi} H^{2}(x+u) d u \int_{0}^{\frac{1}{2} u} \frac{d t}{u^{6}} \leqq A_{\gamma} \int_{2 \alpha \delta}^{\pi} \frac{H^{2}(x+u)}{u^{5}} d u .
$$

Therefore, combining (6.17) and (6.18), it follows that

$$
\begin{aligned}
\int_{0}^{1} \delta & d \delta \int_{0}^{\pi} H^{2}(x+u) d u \int_{0}^{\alpha \delta} \frac{d t}{\left\{\delta^{2}+(t-u)^{2}\right\}^{3}} \\
& \leqq A_{\gamma} \int_{0}^{1} \delta^{-4} d \delta \int_{0}^{2 \alpha \delta} H^{2}(x+u) d x+A_{\gamma} \int_{0}^{1} \delta d \delta \int_{2 \alpha \delta}^{\pi} \frac{H^{2}(x+u)}{u^{5}} d u \\
& =A_{\gamma} \int_{0}^{\pi} H^{2}(x+u) d u \int_{u / 2 \alpha}^{1} \delta^{-4} d \delta+A_{\gamma} \int_{0}^{\pi} \frac{H^{2}(x+u)}{u^{5}} d u \int_{0}^{u / 2 \alpha} \delta d \delta
\end{aligned}
$$




$$
=A_{\gamma} \int_{0}^{\pi} \frac{H^{2}(x+u)}{u^{3}} d u,
$$

from which we conclude that

$$
U^{2}(x ; f) \leqq A_{\gamma} \int_{-\pi}^{\pi} \frac{H^{2}(x+u)}{|u|^{3}} d u
$$

This implies that

$$
U^{2}(x: f) \leqq A_{\gamma} y^{2} \int_{-\pi}^{\pi} \frac{D^{2}(x-u)}{|u|^{3}} d u
$$

since $|H(x)| \leqq 2 y D(x)$ by Lemma 2 .

The remaining part of the proof is quite similar as the preceding one, and we shall omit it here.

7. Some extensions of the integral of Marcinkiewicz. An extension of the lemma of Marcinkiewlcz has been given by Ostrow and Stein [11] as follows: Let $\nu$ be a positive measure on the real axis such that $\nu(E+2 \pi)$ $=\nu(E)$ for any Borel set $E$ and $\frac{1}{t} \int_{0}^{t} d \nu(x) \leqq A(0<t \leqq 2 \pi)$ and the set $P$, $Q$ and the function $D^{\prime}(x)$ be those which are defined at the beginning of $\$ 2$, and $I_{\lambda}(x ; \nu)$ be defined by

$$
I_{\lambda}(x ; \nu)=\int_{-\pi}^{\pi} \frac{D^{\lambda}(x+t)}{|t|^{\lambda+1}} d \nu . \quad \lambda>0 .
$$

Then

$$
\left|\left\{I_{\lambda}(x ; \nu)>s\right\} \cap P\right| \leqq \frac{A_{\lambda, \nu}}{s}|Q| \text {. }
$$

Combining this and the decomposition lemma (Lemma 2), and arguing as in the preceding sections, we have an extention of Theorem 1.

THEOREM 7. Let $\nu$ be a measure described above, and let $f(x) \in L^{1}(-\pi$, $\pi)$, and $\mu(x ; f, \nu)$ be defined by

$$
\mu(x ; f, \nu)=\left\{\int_{0}^{\pi} \frac{[F(x+t)+F(x-t)-2 F(x)]^{2}}{t^{3}} d \nu(t)\right\}^{1 / 2}
$$

where $F(x)=\int_{0}^{x} f(t) d t$. Then

(1) For any $y>0$,

$$
|\{\mu(x ; f, \nu)>y\}| \leqq \frac{A_{\nu}}{y} \int_{-\pi}^{\pi}|f(x)| d x .
$$

(2) The conclusions (2)-(4) of Theorem 1 hold for $\mu(x ; f, \nu)$ instead of $\mu(x ; f)$, and for $A_{p \nu}$ instead of $A_{p}$.

The case $p=2$ of (2) was proved by Ostrow and Stein [11]. 
Also, we can extend Theorem 1 to non-periodic case. Let $f(x)$ be a function integrable on the real axis. Let $\mu(x ; f)$ be defined by

$$
\mu(x ; f)=\left\{\int_{0}^{\infty} \frac{[F(x+t)+F(x-t)-2 F(x)]^{2}}{t^{3}} d t\right\}^{1 / 2} .
$$

Then we have

THEOREM 8. (1)For any $y>0$,

$$
|\{\mu(x ; f)>y\}| \leqq \frac{A}{y} \int_{-\infty}^{\infty}|f(x)| d x .
$$

(2) If $f(x) \in L^{p}(-\infty, \infty), 1<p \leqq 2$, then

$$
\int_{-\infty}^{\infty} \mu^{p}(x ; f) d x \leqq A_{p} \int_{-\infty}^{\infty}|f(x)|^{p} d x
$$

This theorem can be proved in a completely similar manner as in $\S 1$, combining the non-periodic analogues of Lemmas 1 and 2 . Since, however, our main object is not in this extention, we do not enter the details. ${ }^{10)}$

8. The theorem of Paley on Walsh-Fourier series. In this section, we consider only functions and sets periodic with period 1 , and Lemma 1 and Lemma 2 should be transferred to this case.

Let $f(x)$ be integrable in the interval $[0,1]$, and its Walsh-Fourier series be

$$
f(x) \sim \sum_{n=0}^{\infty} c_{n} \psi_{n}(x)
$$

Define :

$$
\delta_{0}(x)=\delta_{0}(x ; f)=c_{0}, \delta_{n}(x)=\delta_{n}(x ; f)=\sum_{j=2^{n-1}}^{2^{n}-1} c_{j} \psi_{j}(x)(n \quad 1)
$$

and

$$
\delta(x)=\delta(x ; f)=\left\{\sum_{n=0}^{\infty} \delta_{n}^{2}(x ; f)\right\}^{1 / 2}
$$

Then the fundamental theorem in the theory of Walsh-Fourier series which is due to R. E. A. C. Paley [12] reads as follows:

THEOREM 9. (1) For $p>1$,

$$
A_{p}\|f\|_{p} \leqq\|\delta(f)\|_{p} \leqq A_{p}\|f\|_{p} .
$$

(2) For any sequence $\left\{\varepsilon_{n}\right\}$ of unit factors and for any $p>1$,

$$
A_{p}\|f\|_{p} \leqq\left\|\sum_{n=0}^{\infty} \varepsilon_{n} \delta_{n}(f)\right\|^{p} \leqq A_{p}\|f\|_{p}
$$
[22].

10) For the extension of the integral of Marcinkiewicz to higher dimensions, see Stein 
The crucial part of the theorem is the right side of (8.4) for $1<p \leqq 2$ or $2 \leqq p<\infty$, and the remaining parts are deduced from this case. For the detailed argument for this fact, we refer the reader to Paley's paper [12].

We shall here give an alternative proof of this theorem by the method developed in the preceding sections.

From the fact above mentioned, it is sufficient to prove the right side inequality for $1<p \leqq 2$, and this is a consequence of the following theorem.

THEOREM 10. For any $y>0$,

$$
|\{\delta(x ; f)>y\}| \leqq \frac{A}{y} \int_{0}^{1}|f(x)| d x .
$$

PROOF. We can restrict ourselves to $f(x) \geqq 0$. We shall first require the explicit formula for $\delta_{n}(x ; f)$. From the property of Walsh functions

$$
\psi_{2^{n}+j}(x)=\psi_{2^{n}}(x) \psi_{j}(x), \quad 0 \leqq j \leqq 2^{n},
$$

hence

$$
\begin{aligned}
\delta_{n+1}(x) & =\sum_{j=2^{n}}^{2^{n+1}-1} c_{j} \psi_{j}(x)=\sum_{j=2^{n}}^{2^{n+1}-1} \psi_{j}(x) \int_{0}^{1} f(t) \psi_{j}(t) d t \\
& =\psi_{2^{n}}(x) \int_{0}^{1} f(t) \psi_{2^{n}}(t) \sum_{j=0}^{2^{n}-1} \psi_{j}(x) \psi_{j}(t) d t .
\end{aligned}
$$

Let

$$
\alpha_{n}(x)=p 2^{-n} \leqq x<(p+1) 2^{-n}=\beta_{n}(x)
$$

for every $x \in[0,1]$, then it is known that

$$
\begin{aligned}
\sum_{j=0}^{2^{n}-1} \psi_{j}(x) \psi_{j}(t) & =2^{n}, \text { for } \alpha_{n}(x) \leqq t<\beta_{n}(x), \\
& =0, \text { elsewhere. }
\end{aligned}
$$

Thus

$$
\delta_{n+1}(x)=2^{n} \psi_{2^{n}}(x) \int_{\alpha_{n}(x)}^{\beta_{n}(x)} f(t) \psi_{2^{n}}(t) d t .
$$

Since $\psi_{2^{n}}(t)=1$ on the left half and $=-1$ on the right half of the interval $\left[\alpha_{n}(x), \beta_{n}(x)\right)$, respectively, it follows that, if we denote the middle point of this interval by $\gamma_{n}(x)$, then

$$
\begin{aligned}
\delta_{n+1}(x) & =2^{n} \psi_{2^{n}}(x)\left\{\int_{\alpha_{n}(x)}^{\gamma_{n}(x)} f(t) d t-\int_{\gamma_{n}(x)}^{\beta_{n}(x)} f(t) d t\right\} \\
& =2^{n} \psi_{2^{n}}(x)\left\{2 F\left(\gamma_{n}(x)\right)-F\left(\alpha_{n}(x)\right)-F\left(\beta_{n}(x)\right)\right\},
\end{aligned}
$$

where $F(x)=\int_{0}^{x} f(t) d t$ 
Hence

$$
\delta(x ; f)=\left[F(1)^{2}+\sum_{n=0}^{\infty} 2^{2 n}\left\{F\left(\alpha_{n}(x)\right)+F\left(\beta_{n}(x)\right)-2 F\left(\gamma_{n}(x)\right)\right\}^{2}\right]^{1 / 2}
$$

Let $F(x)=G(x)+H(x)$ and $f(x)=g(x)+h(x)$ be the decomposition given by Lemma 2 (for the interval $[0,1]$ ), then

$$
\delta(x ; f) \leqq \delta(x ; g)+\delta(x ; h) .
$$

It is known [12] that

$$
\|\delta(g)\|_{2}=\|g\|_{2}
$$

which implies that

$$
y^{2}|\{\delta(x ; g)>y\}| \leqq \int_{0}^{1} g(x)^{2} d x \leqq y \int_{0}^{1} g(x) d x=y \int_{0}^{1} f(x) d x
$$

by (2) and (3) of Lemma 2 , hence

$$
|\{\delta(x ; g)>y\}| \leqq \frac{1}{y} \int_{0}^{1} f(x) d x .
$$

We turn to $\delta(x ; h)$. Let $P$ be the set in Lemma 2 and integrate $\delta^{2}(x ; h)$ over $P_{0}=P \cap[0,1]$, then, since $H(1)=0$ by (4) of Lemma 2 ,

$$
\begin{gathered}
\int_{P_{0}} \delta^{2}(x ; h) d x=\sum_{n=0}^{\infty} 2^{2 n} \int_{P_{0}}\left|H\left(\alpha_{n}(x)\right)+H\left(\beta_{n}(x)\right)-2 H\left(\gamma_{n}(x)\right)\right|^{2} d x \\
\leqq 3 \sum_{n=0}^{\infty} 2^{2 n}\left\{\int_{P_{0}}\left|H\left(\alpha_{n}(x)\right)\right|^{2} d x+\int_{P_{0}}\left|H\left(\beta_{n}(x)\right)\right|^{2} d x\right. \\
\left.+4 \int_{P_{0}}\left|H\left(\gamma_{n}(x)\right)\right|^{2} d x\right\} .
\end{gathered}
$$

Since the arguments are similar for any of sums in the right side of (8.11), we consider the sum

$$
S=\sum_{n=0}^{\infty} 2^{2 n} \int_{P_{0}}\left|H\left(\alpha_{n}(x)\right)\right|^{2} d x
$$

Let $I_{n r}$ denote the interval $r 2^{-n} \leqq x<(r+1) 2^{-n}, 0 \leqq r \leqq 2^{n}$, then $\alpha_{n}(x)$ $=r 2^{-n}$ for $x \in I_{n r}$, so that

$$
\begin{aligned}
S & =\sum_{n=0}^{\infty} 2^{2^{n}} \sum_{r=0}^{2^{n}-1} \int_{P_{0} \cap I_{n r}} H\left(\alpha_{n}(x)\right)^{2} d x \\
& =\sum_{n=0}^{\infty} 2^{2^{n}} \sum_{r=0}^{2^{n}-1} H\left(r 2^{-n}\right)^{2}\left|P_{0} \cap I_{n r}\right| .
\end{aligned}
$$

Since $H\left(r 2^{-n}\right)=0$ if $r 2^{-n} \in P_{0}$, in the inner sum of the last side of (8.12) there remain only terms for which $r 2^{-n} \in \Delta_{j}$ for some $j$, where $\Delta_{j}$ denotes the contiguous interval of $P$. Now, for each $n$, we divide these 
indices $j$ into two classes $A_{n}$ and $B_{n}: j \in A_{n}$ if $\left|\Delta_{j}\right|<2^{-n}$, and $j \in B_{n}$ if $\left|\Delta_{j}\right| \leqq 2^{-n}$. Then

$$
\begin{aligned}
S & =\sum_{n=0}^{\infty} 2^{2 n} \sum_{j \in A_{n}} \sum_{r 2-n_{\epsilon} \Delta,} H\left(r 2^{-n}\right)^{2}\left|P_{0} \cap I_{n r}\right| \\
& +\sum_{n=0}^{\infty} 2^{2 n} \sum_{j \in B^{n}} \sum_{r 2^{-n_{\epsilon}} \Delta_{j}} H\left(r 2^{-n}\right)^{2}\left|P_{0} \cap I_{n r}\right|=T+U,
\end{aligned}
$$

say.

Since, for each $n$, if $\left|\Delta_{j}\right|<2^{-n}$ then there exists at most one index $r$ such that $r 2^{-n} \in \Delta_{j}$, and $D\left(r 2^{-n}\right) \leqq\left|\Delta_{j}\right| \leqq 2^{-n}$ for such $r$ and $j$, it follows by (5) of Lemma 2 that

$$
\begin{aligned}
T & \leqq 4 y^{2} \sum_{n=0}^{\infty} 2^{2 n} \sum_{j \in A_{n}} \sum_{r \cdot 2^{-n_{\epsilon} \Delta_{j}}} D^{2}\left(r \cdot 2^{-n}\right) 2^{-n} \leqq 4 y^{2} \sum_{j}^{\prime}\left|\Delta_{j}\right|^{2} \sum_{2-n>\left|\Delta_{j}\right|} 2^{n} \\
& \leqq 4 y^{2} \sum_{j}^{\prime}\left|\Delta_{j}\right| \leqq A y^{2}|\boldsymbol{Q}|
\end{aligned}
$$

where the prime. indicates that the summation ranges over the indices for which $\Delta_{j}$ is contained in the interval $[-1,2]$.

Next, we turn to $U$. If $I_{n r} \subset \Delta_{j}$ then $P_{0}$ and $I_{n r}$ have no common point, so that

$$
U=\sum_{n=0}^{\infty} 2^{2^{n}} \sum_{j_{\varepsilon} B_{n}} \sum_{r}^{*} H\left(r 2^{-n}\right)^{2}\left|P_{0} \cap I_{n r}\right|,
$$

where $\sum_{r}^{*}$ denote the sum over those $r$ for which $I_{n r}$ intersect with some $\Delta_{j}, j \in B_{n}$, but are not included in it.

Then, for each $n$ and $j$, there exist at most two such indices $r$, and $\left|H\left(r 2^{-n}\right)\right| \leqq 2 y D\left(r 2^{-n}\right) \leqq 2 y 2^{-n}$ for such $r$, so that we get

$$
\begin{aligned}
U & \leqq 4 y^{2} \sum_{n=0}^{\infty} 2^{2 n} \sum_{j \in B_{n}} \sum_{r}^{*} 2^{-2 n} 2^{-n} \\
& \leqq 4 y^{2} \sum_{n=0}^{\infty} 2^{-n} \sum_{\left|\Delta_{j}\right| \geq^{-n}} 2 \\
& =8 y^{2} \sum_{j}^{\prime} \sum_{2^{-n} \leqq\left|\Delta_{j}\right|} 2^{-n} \\
& =A y^{2} \sum_{j}^{\prime}\left|\Delta_{j}\right|=A y^{2}|Q|
\end{aligned}
$$

where the prime has the same meaning as above.

Combining (8.13) and (8.14), and similar ones for other sums in (8.11), we obtain 


$$
\int_{P_{0}} \delta^{2}(x ; h) d x \leqq A y^{2}|Q|
$$

and the proof is completed by the argument as in precedings.

ADDED IN FEBRUARY 12, 1959. After the preperation of this paper, a paper of E. M. Stein, "A maximal function with applications to Fourier series," appeared in Annales of Mathematics, Vol. 68(1958), where he treats the problems analogous to ours. Especially, the results for Cesàro summability of power series of functions of $H^{p}$ class are quite same as ours. The results of Stein for strong summability of Fourier series are incomplete, as he says, since he treats only the case of "index" 2. By the method in our paper, we can complete the results as follows:

THEOREM. Let $f(x) \in L^{p}(-\pi, \pi)$ and $\sigma_{n}^{(\alpha)}(x ; f)=\sigma_{n}^{(\alpha)}(x)$ be the $n$-th Cesâro mean of the Fouries of $f(x)$, and define $T_{q}^{(\alpha)}(x)$ by

$$
T_{q}^{(\alpha)}(x)=\left\{\sup \frac{1}{n+1} \sum_{\nu=0}^{n}\left|\sigma_{\nu}^{(\alpha)}(x)-f(x)\right|^{q}\right\}^{1 / q}
$$

with $1 \leqq p<2, \alpha=1 / p-1,0<q<p /(p-1)$, then

(2) $\left\|T_{q}^{(\alpha)}\right\|_{p} \leqq A_{p, q} \int_{-\pi}^{\pi}|f(x)| \log ^{+}|f(x)| d x+B_{p, q}$ if $f(x) \in L^{p} \log ^{+} L$,

$$
\left\|T_{q}^{(x)}\right\|_{r}^{p} \leqq A_{p, q, r}\|f\|_{p} \quad \text { if } f(x) \in L^{p} \text { and } 0<r<p .
$$

PROOF. Since $p /(p-1)>2$, we can suppose that $q>2$. Let $P$ be the set of points where

$$
\sup _{0<|t| \leqq \pi} \frac{1}{t} \int_{0}^{t}|f(x+u)|^{p} d u \leqq y
$$

and $Q$ be the complement of $P$, then it is proved by Sunouchi*) that for $x \in P$,

$$
T_{q}^{(\alpha)}(x) \leqq A_{q} y^{\alpha+1}\left\{1+\frac{1}{y} \int_{-\pi}^{\pi} \frac{\phi^{q^{\prime}(\alpha+1)-1}(x+t)|f(x+t)|^{p}}{|t|^{q^{\prime} \alpha}} d t\right\}^{1 / q^{\prime}},
$$

where $\phi(x)$ is the function of Marcinkiewicz corresponding to the set $P$ and $1 / q+1 / q^{\prime}=1$ and $q>2$. Hence applying Lemmas 7 and 1 we can conclude that

$$
\left|\left\{T_{q}^{(\alpha)}(x)>A_{p, a} y\right\}\right| \leqq A_{p, q}\left|\left\{M\left(x ;|f|^{p}\right)>y^{p}\right\}\right|,
$$

from which the conclusions of the theorem follow as in the proof of Theorem 3.

*) G. Sunouchi, On the strong summability of power series and Fourier series, Tôhoku Math. J. 6(1954), 220-225. 


\section{BIBLIOGRAPHY}

1. A. P. CALDERón AND A. ZYGMUND, On the existence of certain singular integrals, Acta Math. 88(1952), 85-139.

2. N. J. FINE, Cesàro summability of Walsh-Fourier series, Proc. Nat. Acad. Sci. 41(1955), 588-591.

3. G. H. HARDY AND J.E. LITTLEWOOD, The strong summability of Fourier series, Fund. Math. 25(1935), 162-189.

4. J. E. LitTLEWOOD and R. E. A. C. PALEY, Theorems on Fourier series and power series, Part I. J. Lond. Math. Soc. 6(1931), 230-233 ; Part II, Proc. Lond. Math. Soc. 42(1937), 52-89 ; Part III, ibid, 43(1937), 105-126.

5. N.LUSIN, Sur une propriété des fonction carré sommable, Bull. Calucatta Math. Soc. $20(1930), 139-154$.

6, J. MARCINKIEWICZ, Sur les séries de Fourier, Fund. Math. 27 (1936), 38-69.

7. Sur quelques intégrales du type Dini, Annales de la Soc. Polonaise de Math. 17(1938), 42-50.

8. Sur la sommabilité forte de séries de Fourier, J. Lond. Math. Soc. $14(1939), 162-168$.

9. J. MARCINKIEWICZ AND A.ZYgMund, On a theorem of Lusin, Duke Math. J.4(1938), $473-485$.

10. J. MARCINKIEWICZ, Sur la sommabilité $H_{k}$ de série de Fourier, Comptes Rendus, 208(1939), 782-781.

11. E. M. OstROW AND E. M. STEIN, A generalization of lemma of Marcinkiewicz and Fine, with applications to singular integrals, Annali della Scuola Noim. Sup. di Pisa. 11(1957), 117-135.

12. R. E. A.C.PALEY, A remarkable system of orthogonal functions, Proc. Lond. Math. Soc. (2) $34(1930), 241-279$.

13. G. SUNOUCHI, Note on Fourier analysis (XXXIX), Tôhoku Math. J. (2) 3 (1951). 71-88.

14. G. SUNOUCHI AND S. YANO, On some maximal theorems for strong summability of Fourier series, Math. Japonicae, 2(1951), 53-58.

15. G. SUNOUCHI, Theorems on power series of the class $H^{p}$, Tôhoku Math. J. (2) 8(1956), 125-146.

16. S. YANO, Cesàro summability of Walsh-Fourier series, Tôhoku Math. J. (2) 9(1957), 267-272.

17. A. ZYGMUND, On the convergence and summability of power series on the circle of convergence, Part I, Fund. Math. 30(1938), 170-196; Part II, Proc. Lond. Math. Soc. (2) 47 (1942), $326-350$.

18. - On certain integrals, Trans. Amer. Math. Soc. 55(1944), 170-204.

$19 . \quad$ On a theorem of Marcinkiewicz concerning interpolation of operators, J. Mat'. Pure et Appl. 35(1956), 223-248.

20. On the Littlewood and Paley function $g^{*}(\theta)$, Proc. Nat. Acad. Sci. 42 (1956), 208-212.

21. Trigonometrical series, Warsaw, 1935.

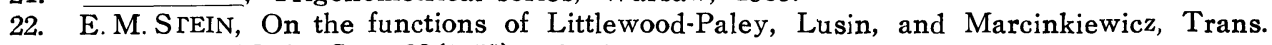
Amer. Math. Soc. 88(1958), 430-466.

Mathematical. Institute, TOKYo MEtropol.tTAN University.

Added in proof July 21, 1959. Since this paper was written, I have learned that the method of this paper was already used in the $2^{\text {nd }}$ eddition of Trigonometric series by Zygmund to prove the existence of conjugate functions (Chapter IV, Theorem (3.16)). 